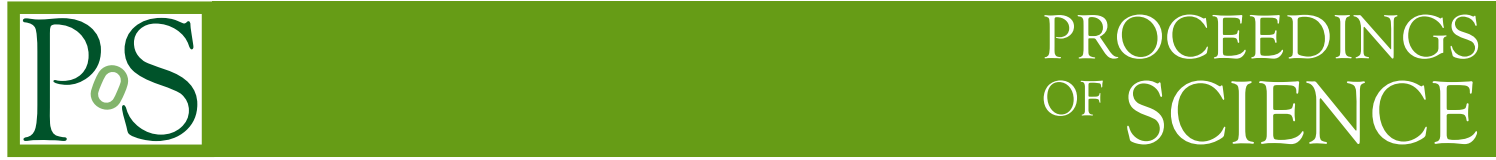

\title{
Searching for Dark Matter and Extra Dimensions at the LHC with intact protons
}

\section{Christophe Royon*}

Department of Physics and Astronomy, The University of Kansas. Lawrence KS 66045, USA

E-mail: christophe.royonaku.edu

After describing the possibilities to detect intact protons in the final state by the CMS-TOTEM and ATLAS experiments at the LHC, we show the sensitivities to quartic anomalous couplings (as a sign for instance of the existence of extra-dimensions in the universe or polarizable dark matter particles) and to axion-like particles.

2nd World Summit: Exploring the Dark Side of the Universe 25-29 June, 2018

University of Antilles, Pointe-à-Pitre, Guadeloupe, France

* Speaker. 
After discussing the possibility of detecting intact protons at the Large Hadron Collider in the CMS-TOTEM and ATLAS experiments, we will describe new methods to look for quartic photon and $W / Z$ bosons anomalous couplings that could be a sign of the existence of extra-dimensions or axion-like particles at high masses. This is definitely complementary to the low-energy experiments that are sensitive to lower masses.

\section{Detecting intact protons in the final state at the LHC}

The ATLAS and CMS-TOTEM collaborations installed some roman pots equiped with Silicon (strips or pixel) and fast timing (quartz, diamond or Silicon) detectors located at about 220 meters from the interaction point [1]. The basic idea is simple: we use tbe magnets from the LHC as a spectrometer in order to scatter at a small angle the protons that lost part of their energy. We detect the intact protons in the AFP (ATLAS Forward Proton) and CT-PPS (CMS-TOTEM Precision Proton Spectrometer) roman pots. The mass acceptance of these detectors depends on how close to the beam the deetctors are inserted, but varies typically betwenn $400 \mathrm{GeV}$ and $2 \mathrm{TeV}$ for standard running at the LHC. Both set of detectors were installed at the end of 2016, and respectively about 15 and $40 \mathrm{fb}^{-1}$ of data have been accumulated in 2016 and 2017. Before the long shutdown starting at the end of $2018,100 \mathrm{fb}^{-1}$ of data should be available.

\section{Looking for $\gamma \gamma \gamma \gamma$ anomalous couplings}

\subsection{Motivation}

Quartic anomalous production of photon pairs can happen via loops of new charged particles or via production of new resonances. We introduce two effective operators at low energies involving the coupling of four photons

$$
L_{4 \gamma}=\zeta_{1}^{\gamma} F_{\mu \nu} F^{\mu v} F_{\rho \sigma} F^{\rho \sigma}+\zeta_{2}^{\gamma} F_{\mu \nu} F^{v \rho} F_{\rho \lambda} F^{\lambda \mu}
$$

In the case of loops of new particles, the effective coupling can be modified as $\zeta_{1}=\alpha_{e m}^{2} Q^{4} m^{-4} N c_{1, s}$ where $m, Q$ and $c_{1, s}$ are respectively the mass, cahrge and spin of the particle in the loop. In the case of a resonance $X$, one gets $\zeta_{1}=\left(f_{s} m\right)^{-2} d_{1, s}$ where $f_{s}$ is the $\gamma \gamma X$ coupling of the new particle to the photon. Typical values of $\zeta_{1}$ are of the order of $10^{-14}-10^{-13} \mathrm{GeV}^{-4}$ for $2 \mathrm{TeV}$ dilatons as an example.

These couplings are motivated by the existence of warped extra-dimensions that solves the hierarchy problem of the Standard Model of particle physics. Typically, our usual 4D brane contains the SM fields whereas gravitation happens via the extra-dimensions.

In the following, all SM or anomalous coupling events were generated using the Forward Physics Monte Carlo (FPMC) [2].

\subsection{Standard model exclusive di-photon production}

In Fig. 1, left are displayed the two different diagrams contributing to the SM exclusive diphoton production, namely the QCD diagram [3] and the QED one. In Fig. 1, right are shown

the contributions of these two diagrams in the exclusive di-photon cross section for a value of 
the di-photon mass larger than the value in abscissa. The QCD contribution drops very fast as a function of the di-photon mass and the QED contribution dominates for masses above $150 \mathrm{GeV}$ (the contributions from quark, leptons and $W$ bosons in the loop are also shown). In the acceptance of the forward proton detectors (masses above $400 \mathrm{GeV}$ ), the only relevant contributions are photoninduced processes. Oberving two photons in CMS or ATLAS and two intact protons in CT-PPS or AFP ensures that this is a photon-induced process $[4,5]$.
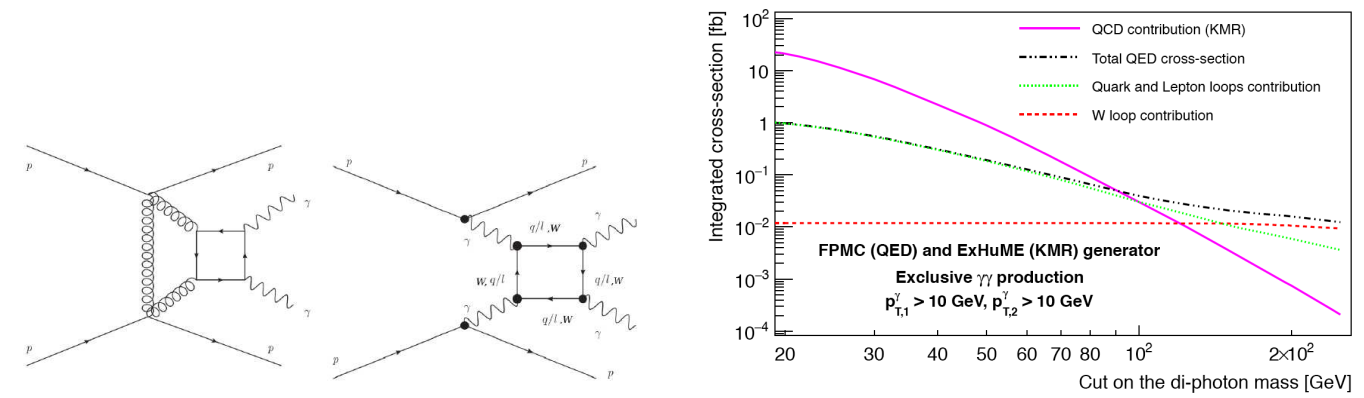

Figure 1: Left: SM diagrams contributing to exclusive di-photon events. Right: Cross section of the different processes for a di-photon mass larger than the value in abscissa.

\subsection{Search for quartic $\gamma \gamma \gamma \gamma$ anomalous couplings}

Quartic anomalous photon couplings might lead to the appearance of two photon and two proton events at high di-photon masses. For a di-photon mass above $600 \mathrm{GeV}$, the exclusive diphoton SM cross section is very small and can be neglected. The only relevant background is due to pile up. At the LHC, bunches of protons collide and in order to look for rare events, many protons collide within the same bunch crossing. Pile up backgrounds correspond in our case as two photons originating from the main hard interactions and intact protons coming from additional soft interactions (additional proton-proton interactions). Additional backgrounds originate from leptons misidentified as photons associated with pile up.

The fact that we detect all particles in the final state for our signal (two photons and two protons) is crucial in order to suppress the pile up background as shown in Fig. 2. We display the ratio of the missing di-proton mass to the di-photon mass (left) and the difference between the diphoton and di-proton rapidities (right). In the case of signal, the two protons system is obviously related to the two photons and this is why the signal peaks at 1.0 or 0.0 in Fig. 2, left and right respectively. For pile up background, the protons are not correlated to the di-photons and this is why, we get a flatter distribution.

The number of signal and background events after each requirement (in the acceptance of the forward detectors, high phton transverse momentum, high di-photon mass, two photons well balanced in transverse momentum and azimuthal angle) for a luminosity of $300 \mathrm{fb}^{-1}$ is shown in Fig. 3. The two last lines of the table show the effect of the requirements to reject pile up events, and less than 0.1 event of background is expected. Any observed event would be a signal event. The expected sensitivity on the anomalous $\gamma \gamma \gamma \gamma$ quartic anomalous coupling is expected to be about $10^{-14} \mathrm{GeV}^{-4}$ reaching the values predicted by some extra-dimension or composite Higgs models 
(we gain about two orders of magnitude on sensitivities with respect to the "standard" methods at the LHC).

Let us note that we used in this study an effective approach but we performed another study using a full amplitude calculation that led to similar results $[4,5]$.
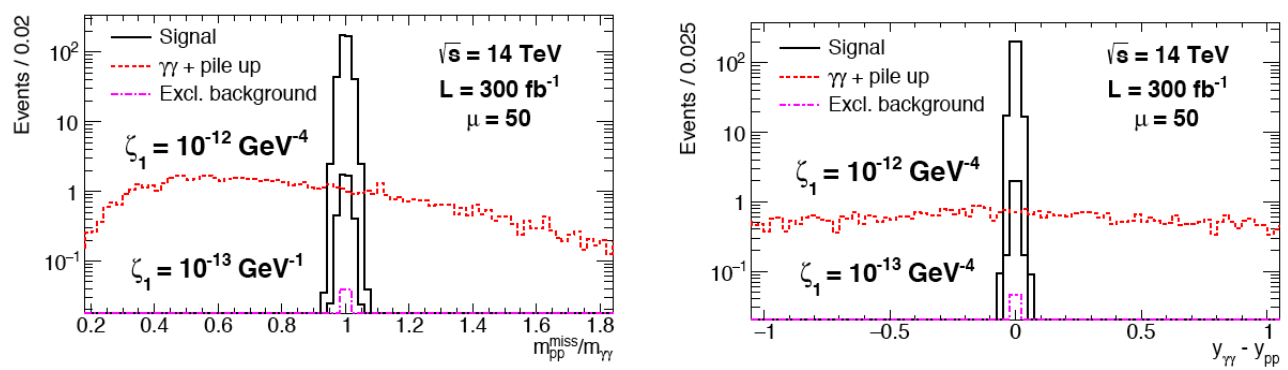

Figure 2: Ratio of the diphoton and di-proton masses and difference in rapidity between the two systems allowing to select signal versus pile up background.

\begin{tabular}{|c||c|c||c|c|c|c|}
\hline Cut / Process & $\begin{array}{c}\text { Signal } \\
\text { (full) }\end{array}$ & $\begin{array}{c}\text { Signal } \\
\text { with (without) } \\
\text { f.f (EFT) }\end{array}$ & Excl. & DPE & $\begin{array}{c}\text { DY, } \\
\text { di-jet } \\
\text { + pile up }\end{array}$ & $\begin{array}{c}\gamma \gamma \\
+ \text { pile up }\end{array}$ \\
\hline \hline $\begin{array}{c}{\left[0.015<\xi_{1,2}<0.15,\right.} \\
\left.p_{\mathrm{T} 1,(2)}>200,(100) \mathrm{GeV}\right]\end{array}$ & 65 & $18(187)$ & 0.13 & 0.2 & 1.6 & 2968 \\
$m_{\gamma \gamma}>600 \mathrm{GeV}$ & 64 & $17(186)$ & 0.10 & 0 & 0.2 & 1023 \\
{$\left[p_{\mathrm{T} 2} / p_{\mathrm{T} 1}>0.95\right.$,} & 64 & $17(186)$ & 0.10 & 0 & 0 & 80.2 \\
$|\Delta \phi|>\pi-0.01]$ & 61 & $16(175)$ & 0.09 & 0 & 0 & 2.8 \\
$\sqrt{\xi_{1} \xi_{2} s}=m_{\gamma \gamma} \pm 3 \%$ & 60 & $12(169)$ & 0.09 & 0 & 0 & 0 \\
$\left|y_{\gamma \gamma}-y_{p p}\right|<0.03$ & 60 & \\
\hline
\end{tabular}

Figure 3: Selection of diphoton signal events with respect to backgrounds.

\subsection{Application to the search for Axion Like Particles at the LHC}

The search for exclusive di-photon production is directly applicable to the search for the Axion Like Particles (ALPs) at the LHC [6]. The ALP is produced as a resonance and decays into two photons. The sensitivity plot (couplings vs mass) is shown in Fig. 4 for a luminosity of $300 \mathrm{fb}^{-1}$. Exclusive di-photon production can increase the sensivity to ALPs at high mass at the LHC and even cover a new domain at very high mass.

It is worth noticing that the same method allows looking for polarizable dark matter candidates with unprecedented precision [7].

\section{Search for quartic anomalous $\gamma \gamma \gamma Z, \gamma \gamma W W$ and $\gamma \gamma Z Z$ anomalous couplings}

Using the same method as described above in order to reject pile up backgrounds, one can look for $\gamma \gamma \gamma Z, \gamma \gamma W W$ and $\gamma \gamma Z Z$ anomalous couplings $[4,8]$. The signals will be $Z \gamma . W W$ or $Z Z$ in the main central detectors and two intact protons in CT-PPS or AFP. In the case of $W W$ exclusive 


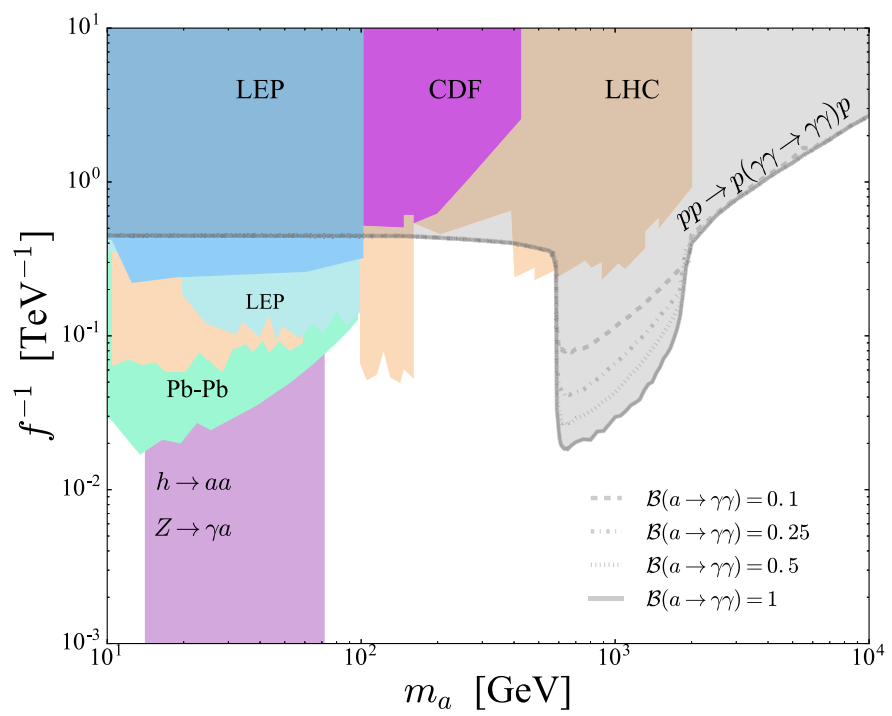

Figure 4: $5 \sigma$ discovery sensitivity on the discovery of ALPS at high mass in the coupling versus mass plane for different assumptions on the branching ratios of ALPs into photons.

production where both $W$ s decay leptonically, the matching method described above does not work because of the presence of neutrinos. Additional detectors can be used in order to measure the time-of-flight of the protons with a typical precision of 15 to 20 picoseconds '[9] and ensure that they originate from the same interaction as the $W \mathrm{~s}$. This additional method allows reducing the pile up background. At the University of Kansas, we take part in developing and testing for instance Ultra-Fast Silicon detectors for CMS-TOTEM and for applications in medicine, chemestry and cosmic-ray experiments. Typical resolutions of 35 ps per layer of Si detectors have been achieved in beam tests.

In the case of $\gamma Z$ production, we studied the cases when the $Z$ boson decays either leptonically or hadronically [8]. In the case when the $Z$ boson decays into hadrons, the matching plot in mass and rapidity are shown in Fig. 5. The distributions are wider than in the di-photon case due to the worse resolution on di-jet mass and rapidity. Combining both decays of the $Z$ bosons allows us to gain about three orders of magnitude on quartic anomalous couplings with respect to more standard methods at the LHC. The studies of $W W$ and $Z Z$ productions when one or two of the $W$ or $Z$ bosons decay hadronically are still in progress and might allow to observe the SM exclusive production of $W$ pairs at high masses.

\section{Conclusion}

In this brief report, we described the search for extra-dimensions, composite Higgs models or polarizable dark particles at the LHC using a new method based on detecting intact protons in the final state. This allows obtaining a negligible background after the event selection and leads to a 

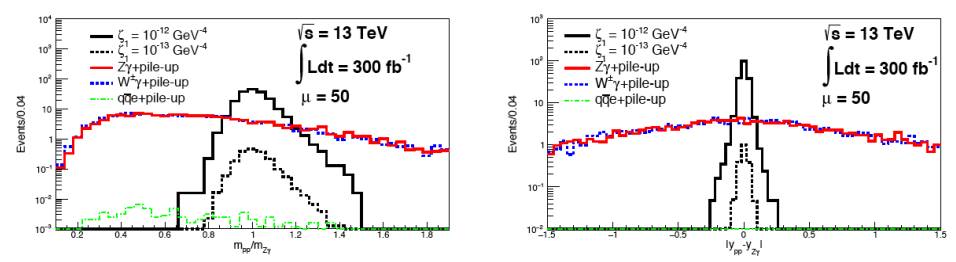

Figure 5: Ratio of the $Z \gamma$ and di-proton masses and difference in rapidity between the two systems allowing to select signal versus pile up background when the $Z$ boson decays hadronically.

better sensitivity by two or three orders of magnitude (depending on the channels) compared to more traditional methods at the LHC.

\section{References}

[1] CMS and TOTEM Coll., CERN-LHCC-2014-021; ATLAS Coll., CERN-LHCC-2015-009.

[2] M. Boonekamp, A. Dechambre, V. Juranek, O. Kepka, M. Rangel, C. Royon, R. Staszewski, e-Print: arXiv:1102.2531; M. Boonekamp, V. Juranek, O. Kepka, C. Royon "Forward Physics Monte Carlo", "Proceedings of the workshop: HERA and the LHC workshop series on the implications of HERA for LHC physics," arXiv:0903.3861 [hep-ph].

[3] V. A. Khoze, A. D. Martin and M. G. Ryskin, Eur. Phys. J. C 23 (2002) 311.

[4] S. Fichet, G. von Gersdorff, B. Lenzi, C. Royon, M. Saimpert, JHEP 1502 (2015) 165; S. Fichet, G. von Gersdorff, O. Kepka, B. Lenzi, C. Royon, M. Saimpert, Phys.Rev. D89 (2014) 114004; E. Chapon, O. Kepka, C. Royon, Phys. Rev. D81 (2010) 074003; O. Kepka and C. Royon, Phys. Rev. D 78 (2008) 073005

[5] S. Fichet, G. von Gersdorff, C. Royon, Phys. Rev. Lett. 116 (2016) no. 23, 231801; S. Fichet, G. von Gersdorff, C. Royon, Phys. Rev. D 93 (2016) no. 7, 075031.

[6] C. Baldenegro, S. Fichet, G. von Gersdorff, C. Royon, JHEP 1806 (2018) 131

[7] S. Fichet, JHEP 1704 (2017) 088.

[8] C. Baldenegro, S. Fichet, G. von Gersdorff, C. Royon, JHEP 1706 (2017) 142.

[9] N. Minafra et al., Nucl. Instrum. Meth. A867 (2017) 88; D. Breton et al., Nucl. Instrum. Meth. A835 (2016) 51; Nucl. Instrum. Meth. A895 (2018) 158. 